\title{
Aplicaciones de técnicas físico-químicas en Antropología Forense
}

\author{
The application of physico-chemical techniques to \\ Forensic Anthropology
}

\begin{abstract}
Resumen
Los efectos del calor extremo sobre los restos óseos es un tema de gran interés para el mundo forense. La literatura en rápido crecimiento en esta área de las ciencias forenses incluye investigaciones experimentales que analizan la dinámica del impacto térmico sobre la estructura del esqueleto y su morfología. Además de los métodos tradicionales de investigación, la aplicación de técnicas químico-físicas como la difracción de rayos X (DRX) y la espectroscopia de infrarrojos por transformada de Fourier (FT-IR) es cada vez más aceptada en distintos contextos forenses. Los campos de aplicación se refieren principalemente a la capacidad para distinguir entre restos humanos quemados y otros materiales, la determinación de la temperatura, el tiempo de combustión y su intensidad en todo el cuerpo, en diversas situaciones como accidentes, suicidios/homicidios y el estudio de la escena del crimen.
\end{abstract}

Palabras clave: Huesos quemados. DRX. FT-IR. Índice de cristalinidad. Temperatura.

\section{Abstract}

The effects of extreme heat on the skeletal remains are a subject of great interest to the forensic world. The rapidly growing literature in this area of forensic science includes experimental investigations that analyze dynamics of thermal impact on the skeletal structure and morphology.

In addition to traditional research methods, the application of chemical and physical techniques such as X-ray diffraction (XRD) and, Fourier transform infrared spectroscopy (FT-IR) is increasingly accepted in forensic contexts. The ability to distinguish between burned human remains and other materials, and the determination of the temperature, the combustion time and intensity throughout the body may be important in various situations such as accidents, suicides / homicides and studying the crime scene.

Key words: Burned bones. XDR. FT-IR. Cristallinity index. Temperature.

\section{Introducción}

El estudio de los restos humanos quemados es de gran importancia en la arqueología, las ciencias forenses, la antropología forense y la investigación de la escena del crimen. En el campo de las ciencias forenses, existen una gran variedad de situaciones que pueden conllevar la cremación de restos óseos, incluyendo los accidentes de aviación, los bombardeos, las explosiones y terremotos. También los homicidios, suicidios y muertes accidentales pueden implicar el uso de fuego con resultados variables sobre los restos humanos.
El fuego es un método común para tratar de ocultar la evidencia de la actividad criminal infligida sobre víctimas humanas. El fuego puede ser utilizado por el autor para: 1) destruir totalmente el cuerpo, 2) destruir las características que permiten la identificación de víctimas (rasgos faciales, huellas dactilares), o 3 ) destruir pruebas relacionadas con las circunstancias que rodean la muerte ${ }^{1}$. El deterioro o la desaparición de los tejidos blandos por el fuego dificulta notablemente o impide totalmente el análisis por otros especialistas (como los médicos forenses) y, por tanto, el análisis de los restos humanos quemados es una tarea comúnmente atribuida a los antropólogos forenses.
G. Piga ${ }^{1,2}$

A. Malgosa ${ }^{1}$

${ }^{1}$ GROB (Grup de Recerca en OsteoBiografia). Unitat d'Antropologia Biològica, Departament de Biologia Animal, Biologia Vegetal i Ecologia, Universitat Autònoma de Barcelona, España. 2Dipartimento di Chimica e Farmacia, Università di Sassari. Italia.

Correspondencia: Dra. Assumpció Malgosa. E-mail: assumpcio.malgosa @uab.cat.

Fecha de recepción: 17.MAR.2012

Fecha de aceptación: 23.MAR.2012 
Por otro lado, también las muestras arqueológicas pueden presentar evidencias de incineración y su análisis proporciona datos relevantes para reconstruir el ritual funerario y por tanto para entender cuestiones trascendentales de las poblaciones del pasado 2,3 .

Así pues, la comprensión de los cambios que el cuerpo ha sufrido como consecuencia de su exposición al fuego puede proporcionar información importante sobre el contexto y las condiciones del evento de cremación. Dicha información puede incluir la escena del crimen, la temperatura del fuego, y la presencia de acelerantes. En este sentido, se dispone de información sobre la temperatura que alcanzan algunos combustibles al arder al aire libre: por ejemplo, la grasa animal a $800-900^{\circ} \mathrm{C}$, el queroseno a $990^{\circ} \mathrm{C}$, la gasolina a $1.026^{\circ} \mathrm{C}$, la madera a $1.027^{\circ} \mathrm{C}$ y el metanol a $1.200^{\circ} \mathrm{C}^{4}$.

Puesto que la cremación puede conducir a la fragmentación extrema del hueso, se requiere una especial atención durante el análisis de la escena. Los escenarios de incendios mortales suelen ser mucho más complejos, no sólo porque el cuerpo y los elementos de identificación son drásticamente modificados por el fuego, sino porque todo el entorno, todo el contexto, también se modifica en la misma manera, resultando en una coloración homogénea y en la mezcla de todos los materiales.

Idealmente, los antropólogos forenses deberían participar en la recuperación, ya que están capacitados para reconocer los restos humanos fragmentados. Aún así, puede ser un reto, incluso para antropólogos entrenados, el distinguir pequeños fragmentos de huesos quemados y dientes a partir de fragmentos carbonizados de materiales de construcción y otros.

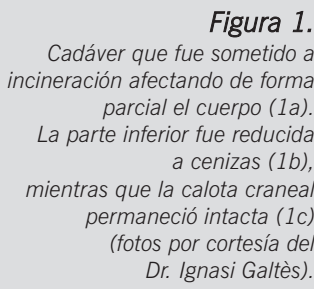

Figura 1 Cadáver que fue sometido a incineración afectando de forma parcial el cuerpo (1a). La parte inferior fue reducida a cenizas ( $1 b)$ mientras que la calota craneal permaneció intacta (1c) (fotos por cortesía de Dr. Ignasi Galtès)
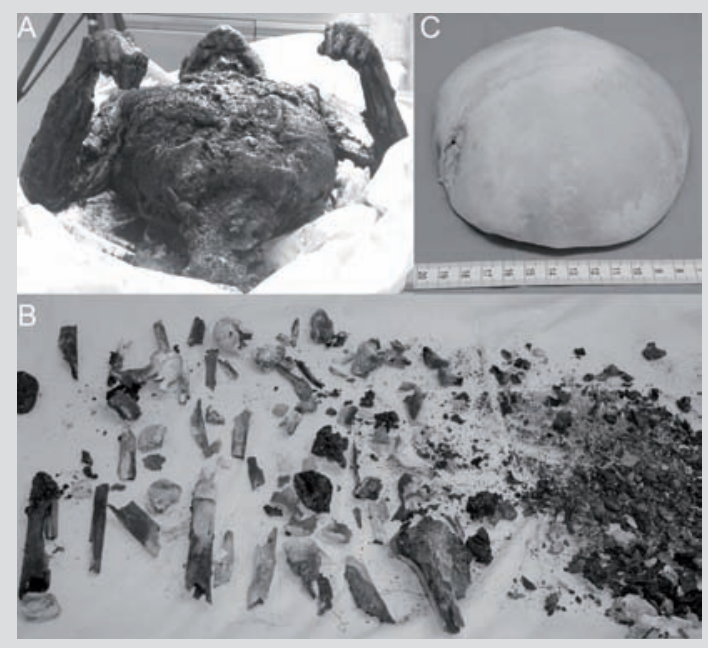

Por otro lado, la capacidad de discriminar entre restos humanos quemados (cenizas) y polvo de otros materiales de apariencia similar puede ser de gran importancia en una variedad de situaciones. El incidente de Tri-State en Noble (Georgia, Estados Unidos) $)^{5}$ es el ejemplo más conocido. A principios de 2002, se descubrió que en lugar de realizar las incineraciones contratadas, el propietario de Tri-State enterraba los cuerpos alrededor de su propiedad, sin ningún tipo de ceremonia. Con el tiempo se recuperaron más de 330 cuerpos, mientras que las urnas que habían recibido muchas familias a menudo contenían polvo de cemento, sílice, piedra y otros materiales. La confusión fue enorme ya que la mayoría de los cuerpos recibidos antes de cierta fecha fueron realmente incinerados, y más tarde, algunos cuerpos podrían haber sido enviados a otras instalaciones para una incineración apropiada. Cientos de familias no estaban seguros del contenido de las urnas en su poder. Así pues, incluso las cremaciones comerciales presentan problemas para el médico forense y las disputas sobre las cremaciones comerciales pueden desembocar en un litigio civil que puede implicar el análisis forense de los materiales recuperados.

El análisis de los restos resultantes de la cremación comercial incluye también la evaluación del número mínimo de individuos y de las posibles mezclas, así como la identificación. Frecuentemente la recuperación y el análisis de inclusiones, tales como placas metálicas de identificación, restauraciones dentales y los materiales quirúrgicos facilitan las identificaciones.

Por desgracia, el acto de quemar aunque no llegue a la incineración total provoca una serie de cambios sustanciales en el cuerpo y en el esqueleto, que a su vez puede afectar los intentos de proporcionar la identificación de los fallecidos. La investigación ha demostrado que tanto los métodos morfológicos y métricos de evaluación antropológica como los métodos de datación, elementos traza ${ }^{6}$ y análisis de isótopos estables, se ven afectados.

Tradicionalmente se ha utilizado la inspección visual de los restos para discriminar si los huesos han sido sometidos al fuego, y más allá de esto, asociaciones entre el color del hueso y el tipo de fractura con la temperatura del fuego, o con la presencia o ausencia de tejidos blandos en el momento de la exposición ${ }^{7,8}$.

Sin embargo, estas asociaciones son complejas, no totalmente biunívocas, y los vínculos espurios. Por ejemplo, en algunos casos de homicidio en los que el fuego fue usado para encubrir las pruebas, los cuerpos fueron sometidos a diversos grados de combustión (Figura 1a y 1b); la observación del color de 
las diversas partes del esqueleto como única referencia, podría haber proporcionado una información inexacta o incompleta en la que incluso se puede excluir la presencia de fuego en alguna parte del esqueleto (Figura 1c).

También se ha demostrado experimental y estadísticamente que los cambios más importantes en el hueso que pueden predecir el contexto de una cremación implican cambios en la microestructura ósea ${ }^{9}$. Por ello, se ha argumentado que la mejor forma, y la más confiable, para abordar los problemas relacionados con la exposición al fuego es la utilización de métodos físico-químicos, posiblemente en combinación con otros tipos de métodos microscópicos, dedicando una atención especial a la fase mineral de la hidroxiapatita (HA), que es el principal componente inorgánico del esqueleto.

Las técnicas de difracción de rayos $X(X R D)$ y la espectroscopia de infrarrojos (FT-IR) se pueden utilizar para distinguir los materiales óseos de otros tipos de materiales cuando el contexto forense no está claro, y para evaluar la estructura cristalina del hueso quemado, lo que, a su vez, se puede relacionar con la temperatura y la intensidad de la cremación.

\section{Métodos químicos-físicos para el análisis de cremaciones}

La medida del índice de cristalinidad (IC) se ha utilizado para estudiar los cambios en la microestructura del hueso. EI IC es una medida del orden existente dentro del cristal, de la deformación y la organización en el hueso. Cuando el hueso es fresco, la estructura química es poco cristalina ${ }^{10,11}$, en parte como resultado de la sustitución de carbonatos por fosfatos causando un desorden del cristal ${ }^{12}$.

La cristalinidad no es uniforme en todo el esqueleto, y varía entre los diferentes tejidos mineralizados del cuerpo humano ${ }^{13}$. También se ha demostrado que la edad afecta la cristalinidad, ya que a menor edad el hueso es menos cristalino que un hueso maduro ${ }^{10}$.

Como resultado de la acción del calor sobre el hueso y su posterior cremación, la estructura cristalina de hueso se vuelve más ordenada y se caracteriza por cristales más grandes, con lo que aumenta el valor del IC. Por ello, el IC ha sido utilizado para estudiar el mineral óseo calentado en un gran número de contextos; diversos ejemplos incluyen el uso del índice de cristalinidad para determinar si el hueso fue quemado o no ${ }^{14}$ y para observar la existencia de diferencias en las prácticas funerarias ${ }^{3,15}$.
El cálculo del IC se puede realizar tanto mediante la difracción de rayos X (DRX), como con la espectroscopía infrarroja con transformada de Fourier (FTIR), aunque los cálculos específicos y los valores serán diferentes entre ambos.

\section{Difracción de rayos $\mathrm{X}(\mathrm{DRX})$}

La espectrometría de Difracción de rayos $X$ (DRX) es una de las herramientas analíticas más potentes para la identificación de sustancias cristalinas desconocidas. Tiene varias ventajas sobre otras técnicas analíticas para la identificación de los restos cremados ya que no es destructiva, requiere cantidades relativamente pequeñas de material (aproximadamente $0,5 \mathrm{gr}$ ), no se ve afectado por las variaciones elementales presentes en la bioapatita, y se puede utilizar para el cálculo semi-cuantitativo de los componentes de una mezcla, determinando así el nivel relativo de contaminación de una muestra.

El método se basa en el hecho de que todos los cristales están compuestos por planos regulares y repetitivos de átomos que forman un retículo. Cuando los rayos $X$ coherentes se dirigen a un cristal, los rayos- $X$ interactúan con cada átomo en el cristal, excitando los electrones y haciéndolos vibrar con la frecuencia de la radiación incidente. Los electrones se convierten en fuentes secundarias de rayos $X$, re-irradiando esta energía en todas las direcciones en la misma longitud de onda del haz incidente, un fenómeno conocido como dispersión coherente. Estos rayos $\mathrm{X}$ difractados se pueden comparar con las ondas que viajan en todas direcciones y forman patrones de interferencia muy similares a las interferencias que se forman cuando se dejan caer dos piedras en el agua. Esta interferencia puede ser constructiva, formando olas mayores, o destructiva, anulando totalmente las olas. El patrón de interferencia creado depende de la distancia entre las capas atómicas, la composición química, y el ángulo en que los rayos $X$ difractan lejos de los átomos, por lo que indirectamente revela la estructura de los cristales.

En el espectrómetro de difracción de rayos $X$, el tubo de rayos $X$ y el detector se hacen girar alrededor de la muestra. El espectro de difracción creado por interferencia constructiva es registrado por el detector del haz. La relación entre el ángulo en el que se producen los picos de difracción y la distancia entre los átomos de una red cristalina (distancia interplanar d) se expresa por la ley de Bragg: $\mathbf{n l}=\mathbf{2} \mathbf{d}$ sinq.

Por razones históricas, los difractogramas se expresan en grados dos theta $(2 \theta)$. Los espectros de difracción se recogen en el rango angular de $9^{\circ}$ a $140^{\circ}$ en $2 \theta$ y 
se analizan con un software llamado MAUD (Material Analysis Using Diffraction) ${ }^{16}$, que permite corregir los datos para la función instrumental y evaluar cuantitativamente las fases mineralógicas presentes en los huesos junto con los parámetros reticulares y microestructurales. Se requieren de 12 a 24 horas para recoger un espectro.

Puesto que cada sustancia tiene una estructura cristalina única (fase cristalográfica), los ángulos de interferencia constructiva forman un patrón único. Al comparar las posiciones e intensidades de los picos de difracción con una biblioteca de materiales cristalinos conocidos, se puede identificar la composición de las muestras de la fase desconocida, como si fuera la huella digital de la substancia. Esto es cierto también para una mezcla de sustancias. En la práctica, la mezcla de 5-6 fases cristalográficas se pueden resolver con un grado de confianza aceptable.

En 1975 se demostró que las altas temperaturas que se consiguen con el tratamiento con fuego inducen un aumento de las dimensiones medias de los microcristales de la apatita, que se puede medir a partir del alargamiento/restricción de los picos de difracción ${ }^{17}$.

A fin de afrontar la problemática relativa al estudio de restos humanos arqueológicos quemados o presuntamente quemados, en nuestro laboratorio efectuamos la calibración de una muestra de hueso que se utilizó como referencia. Para ello se simuló la exposición al fuego real en función de distintas temperaturas de tratamiento controlado $\left(200-1100^{\circ} \mathrm{C}\right)$, tomando en consideración la dependencia del tiempo $(0,18 \text { y } 60 \text { minutos })^{14}$.

En particular se analizó el comportamiento del hueso de referencia a determinadas temperaturas intermedias $\left(650,750,775,825,850^{\circ} \mathrm{C}\right)$ a fin de monitorizar y explicar el doble régimen de crecimiento en los cristales de la hidroxiapatita en el que se aprecia un aumento súbito entorno a los $700^{\circ} \mathrm{C}$, acercándose luego a un valor constante más alto, siguiendo un comportamiento sigmoidal con un tipo de función logística ${ }^{14}$. Este intervalo de temperatura es importante porque durante esta transición el tamaño de los cristales de los huesos se altera a un grado estadísticamente significativo ${ }^{9,18}$.

Asimismo, se puede cuantificar la cinética del crecimiento de los cristales en relación a la temperatura y al tiempo de tratamiento, para disponer de un punto inmediato de referencia en la aplicación de la calibración de los restos presuntamente quemados. De esta forma, se puede no sólo determinar con más exactitud la temperatura alcanzada por los restos, sino que también es posible hacer una aproximación al tiempo de cremación.
Estos datos tienen sus limitaciones ya que debe tenerse en cuenta que se ha trabajado sobre hueso seco. A pesar de ello, Bohnert y colaboradores ${ }^{19}$ han observado que para la total incineración de un cuerpo a través de la cremación se precisan cerca de dos horas a una temperatura de $800{ }^{\circ} \mathrm{C}$, mientras que para la destrucción de las partes blandas son necesarios al menos 50 minutos. Así pues los tiempos de estudio programados en nuestro laboratorio son coherentes con una cremación real.

La Figura 2 muestra el espectro de difracción del hueso humano no quemado usado como referencia. Los puntos se refieren a los datos experimentales, mientras la línea continua es la aproximación (fit) conseguida con el método de Rietveld después del ajuste iterativo de los parámetros estructurales y microestructurales. Los picos de difracción de la hidroxiapatita resultan extremadamente alargados debido a la naturaleza nanocristalina del material óseo que comporta dimensiones medias de los cristales extremamente reducidas y una elevada concentración de desorden reticular. Después de haber tenido en cuenta los efectos instrumentales, el método de Rietveld es capaz de distinguir y separar el alargamiento de los picos de difracción en términos de dimensiones medias de los cristales y de densidad del desorden reticular, expresadas en Angstroms $\left(1 \AA=10^{-8} \mathrm{~cm}\right)$. En particular, la dimensión media de los cristales resulta ser de $170( \pm 5) \AA$.

Así pues estos valores pueden ser considerados representativos de un hueso no quemado.

Las temperaturas de 200 hasta a $600{ }^{\circ} \mathrm{C}(0$ minutos) muestran procesos de crecimiento de los cristales muy débiles. El calentamiento isocrono de la muestra (velocidad $20^{\circ} \mathrm{C} / \mathrm{min}$ ) seguida de un calentamiento isotermo a las temperaturas establecidas (18, 36 e 60 minutos) parece tener efectos limitantes sobre el crecimiento de los cristales debido a una relativa estabilidad del sistema. Contrariamente, es evidente un efecto de crecimiento rápido de los cristales a temperaturas superiores a $700{ }^{\circ} \mathrm{C}(60 \mathrm{~min})$, ulteriormente distinguible en el intervalo de temperatura entre $750^{\circ}$ y $850^{\circ} \mathrm{C}$, y observable en todos los tiempos en los que se ha actuado ( $0,18,36$ y $60 \mathrm{~min})$.

En lo concerniente a los huesos tratados a temperaturas mayores de $850{ }^{\circ} \mathrm{C}$ y que han permanecido largamente en el horno, las dimensiones de los cristales son superiores a $1.500 \AA$ aunque la determinación de su valor medio es bastante difícil ya que se sitúa en el límite instrumental de la técnica.

Para temperaturas superiores a $1.000^{\circ} \mathrm{C}$, las dimensiones medias de los cristales parecen aproximarse a los valores máximos asintóticos siguiendo un proceso de crecimiento de tipo sigmoidal (Tabla 1$)^{14}$. 


\begin{tabular}{|c|c|c|c|c|}
\hline Temperatura $/{ }^{\circ} \mathrm{C}$ & 0 Minutos & 18 Minutos & 36 Minutos & 60 Minutos \\
\hline No quemado & 170 & & & \\
\hline 200 & 175 & 175 & 175 & 175 \\
\hline 300 & 180 & 184 & 186 & 188 \\
\hline 400 & 195 & 203 & 204 & 205 \\
\hline 500 & 202 & 202 & 205 & 210 \\
\hline 600 & 204 & 226 & 230 & 256 \\
\hline 650 & 213 & 240 & 250 & 258 \\
\hline 700 & 229 & 294 & 463 & 486 \\
\hline 750 & 268 & 611 & 712 & 800 \\
\hline 775 & 350 & 836 & 880 & 920 \\
\hline 800 & 432 & 1030 & 1160 & 1200 \\
\hline 825 & 732 & 1120 & 1140 & 1254 \\
\hline 850 & 923 & 1380 & 1450 & 1500 \\
\hline 900 & 1.351 & $>1.500(1616)$ & $>1.500(1680)$ & $>1.500(2621)$ \\
\hline 1000 & $>1.500(1569)$ & $>1.500(2195)$ & $>1.500(2600)$ & $>1.500(2950)$ \\
\hline
\end{tabular}

Tabla 1.

Tamaño medio de cristales de la fase mineral hidroxiapatita (1 $\AA=10-8 \mathrm{~cm})$

(de Piga et al, 2009).
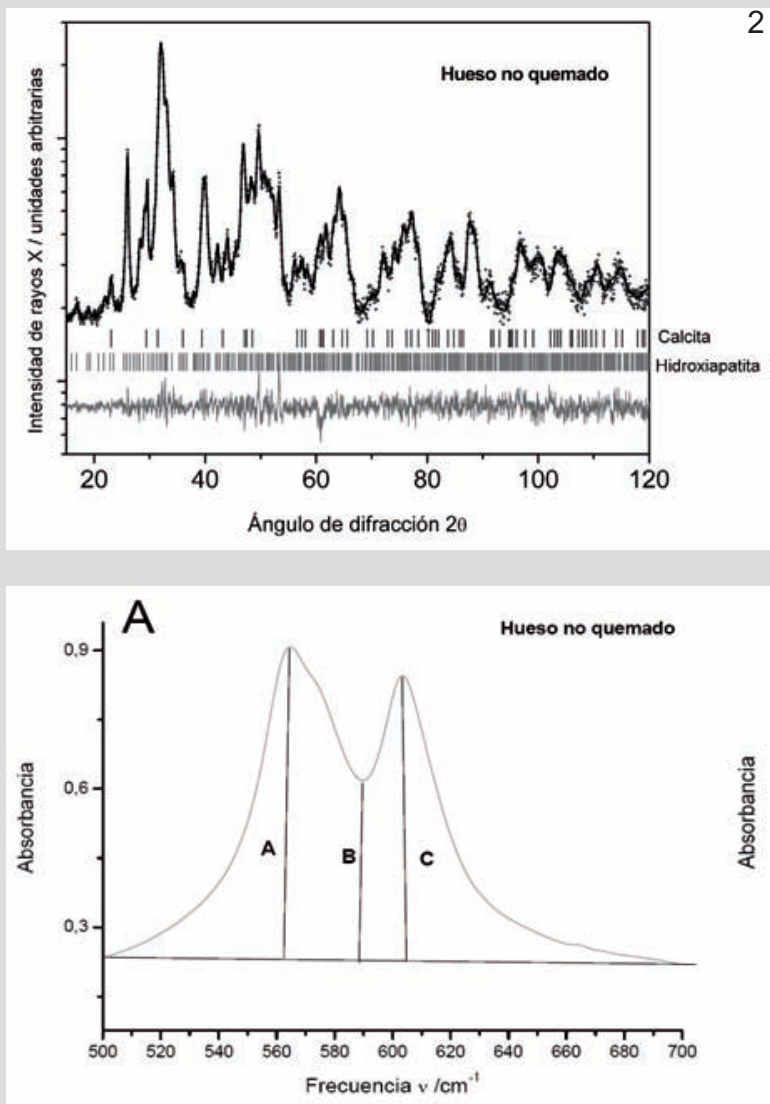
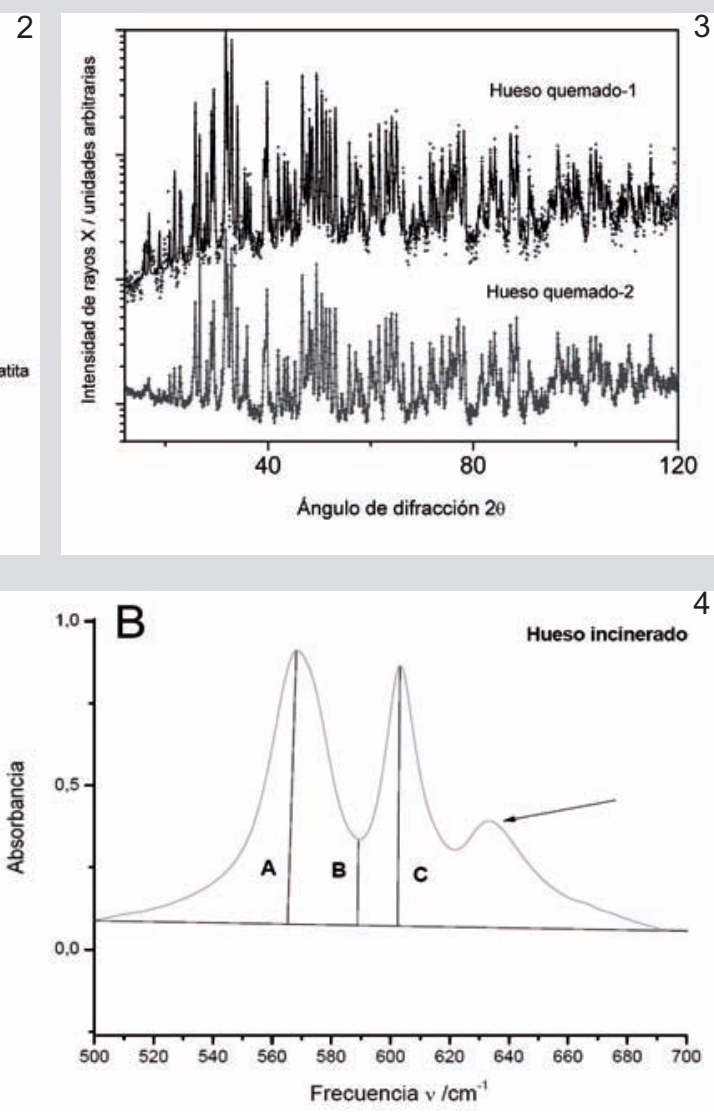

Figura 2.

Patrón de DRX de hueso humano no tratado usado como patrón de referencia. La línea con puntos muestra los datos experimentales, la línea continua es el ajuste de Rietveld en base a la estructura de la hydroxiapatita y la calcita. La línea inferior se refiere a los valores residuales; es decir la diferencia entre la raíz cuadrada de las intensidades calculadas y obtenidas, la cual es indicativa de la bondad de los resultados.

Figura 3.

Difractogramas de dos huesos tratados a elevadas temperaturas $\left(1.000^{\circ} \mathrm{C}\right)$, en los que es evidente una considerable agudización y estrechamiento de los picos de rayos $X$.

4 Figura 4a.

Espectro de FT-IR de un hueso no quemado, mostrado en la gama de $500-700 \mathrm{~cm}^{-1}$, correspondiente al grupo $\mathrm{PO}_{4}{ }^{3-}$ característico de la hidroxiapatita. El factor de dimensiones SF se calcula numéricamente en este grupo de picos.

Figura $4 b$.

Espectro de la banda de los fosfatos de un hueso quemado. La anchura de las dos bandas en 565 y $605 \mathrm{~cm}^{-1}$ disminuye a medida que aumenta la

temperatura, con la aparición simultánea de un tercer pico a aproximadamente $630 \mathrm{~cm}^{-1}$ 
En las dos muestras incineradas mostradas en la Figura 3 se puede observar la notable reducción de los picos de difracción, debido al notable crecimiento de los microcristales y a la reducción del desorden reticular inducido por el considerable tratamiento térmico $\left(1000{ }^{\circ} \mathrm{C}\right)$ al que fueron sometidos.

\section{FTIR}

Para apoyar e integrar los resultados obtenidos también se ha utilizado la técnica de espectroscopia de Infrarrojos por transformada de Fourier (FT-IR), un diagnóstico ampliamente utilizado en la químicafísica que permite reconocer la presencia de grupos o especies moleculares característicos de las muestras sometidas a examen.

Contrariamente a la difracción de rayos $\mathrm{X}$, esta medida tiene la ventaja de usar cantidades de muestra del orden de unos pocos miligramos (aproximadamente 0,003 gr) y requerir tiempos de adquisición del espectro muy cortos (aproximadamente 50 segundos). El espectro característico de los grupos moleculares está determinado por las frecuencias de absorción específicas de los movimientos de rotación, flexión o "estiramiento" (que consiste en la contracción y expansión de las distancias entre los átomos).

Con respecto al material óseo humano y animal, por lo general se analizan las características del banda de fosfatos $\left(\mathrm{PO}_{4}{ }^{3-}\right)$, detectables en la gama de frecuencias entre $500 \mathrm{~cm}^{-1}$ y $700 \mathrm{~cm}^{-1}$.

Como puede verse en la Figura $4 a$, la banda de fosfatos en el material óseo no quemado está compuesto de al menos dos componentes, que son lo suficientemente grandes, pero que disminuyen a medida que aumenta la temperatura, con la consiguiente aparición de otro pico a aproximadamente $630 \mathrm{~cm}^{-1}$ (Figura 4b). El estrechamiento de estos dos componentes se puede medir numéricamente con una buena precisión utilizando un índice de cristalinidad llamado Splitting factor SF ("factor de división").

El índice de cristalinidad SF se calcula por $(A+C) / B)$, donde $A, B$ y $C$ representan la distancia a la línea de base. Así pues, en la línea de base del espectro, se suman las alturas de las absorciones en aproximadamente 605 y $565 \mathrm{~cm}^{-1}$ y se divide por la altura mínima entre ellos (en $595 \mathrm{~cm}^{-1}$ ).

Esta fórmula fue propuesta por primera vez por Shemesh $^{20}$ y Weiner y Bar-Yosef ${ }^{21}$ y ha sido habitualmente utilizado desde entonces.

Las longitudes de onda en $565 \mathrm{~cm}^{-1}$ y $605 \mathrm{~cm}^{-1} \mathrm{co}$ rresponden a las bandas de vibración de flexión de fosfatos y se incrementan con el aumento de la cristalinidad. La longitud de onda en $595 \mathrm{~cm}^{-1}$ disminuye provocando así un aumento global en el valor de SF. Nótese que aunque el aumento de temperatura provoca un aumento de el SF, la relación no es lineal sino que sigmoidal ${ }^{22}$.

Las bandas con mayor frecuencia indican la presencia de grupos carbonatos $\left(\mathrm{CO}_{3}{ }^{2-}\right)$ que proporcionan informaciones adicionales útiles de características químico-físicas sobre el hueso.

Una estimación del contenido de carbonato viene dada por la relación de la absorción del pico a 1428 $\mathrm{cm}^{-1}\left(\mathrm{CO}_{3}{ }^{2-}\right)$ y del pico a $1042 \mathrm{~cm}^{-1}\left(\mathrm{PO}_{4}{ }^{3-}\right)$; se indica como C/P. El valor de este índice disminuye cuando aumenta la temperatura.

El uso combinado de ambas técnicas (DRX y FITR) constituye una poderosa herramienta para evaluar si los huesos se han sometido al fuego y a qué temperatura, con una gran fiabilidad ${ }^{22}$.

\section{Aplicaciones}

Existen casos en los que un tratamiento térmico no es particularmente evidente, lo que comportaría quizás una línea errónea en la investigación de los hechos. Para ilustrar estos casos utilizamos el caso arqueológico de la necrópolis fenicia y púnica de Monte Sirai (Carbonia, Cerdeña-Italia) ${ }^{22}$ en la que la cremación se utilizó como rito funerario durante la época fenicia, mientras que la época púnica se caracteriza por la inhumación de los cadáveres.

Sin embargo, algunos esqueletos de época púnica mostraron algunas trazas oscuras sobre los huesos aunque el conjunto esquelético se presentaba completo y en posición anatómica (Figura 5). Los antecedentes de cremación en la población fenicia nos indujeron a realizar un análisis físico-químico sobre los restos.

Mientras el espectro DRX relativo al esqueleto de la Tumba 252 (Figura 6) muestra inequívocamente las características de una incineración -rito en uso por parte de la población fenicia hasta el siglo VI a.C., los datos relativos a los individuos de la tumba 8 , 12 e 255 muestran también una exposición al fuego, aunque de menor intensidad. Este hecho está en principio en desacuerdo con lo que se podría esperar en el caso de tumbas púnicas, época posterior a la fenicia y cuyo rito generalizado es la inhumación. Los resultados obtenidos con ambas técnicas se muestran en la Tabla 2. 


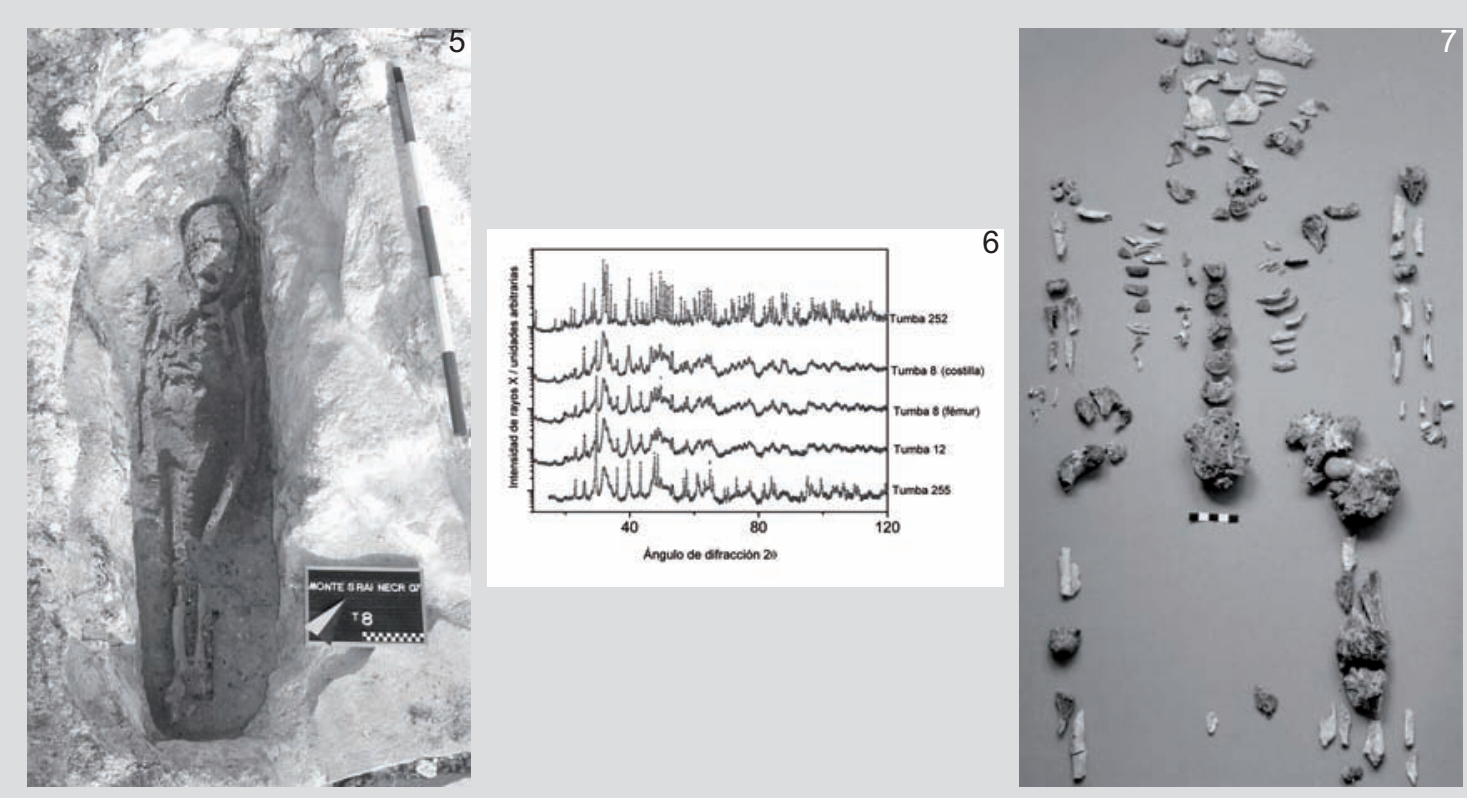

Figura 5.

Restos esquelético de la tumba $n^{\circ} 8$ de época púnica del

yacimiento sardo de Monte Sirai.

Figura 6.

Patrones de los espectros de DRX de 5 muestras procedentes del yacimiento sardo de Monte Sirai.

Figura 7.

Reconstrucción en el laboratorio del esqueleto procedente de la tumba 252 conservado de manera excepcional.

\begin{tabular}{|l|c|c|c|c|}
\hline Muestra & $\begin{array}{c}\text { Tamaño medio de los } \\
\text { cristales } / \AA\end{array}$ & $\begin{array}{c}\text { Temperatura } \\
\text { DRX } /{ }^{\circ} \mathrm{C}\end{array}$ & Splitting Factor (SF) & $\begin{array}{c}\text { Temperatura } /{ }^{\circ} \mathrm{C} \\
\text { FT-IR }\end{array}$ \\
\hline Tumba 252 & $>1500(2297)$ & 1000 & 6,18 & $>900$ \\
\hline Tumba 8-1 & 251 & $\cong 650$ & 4.20 & 695 \\
\hline Tumba 8-2 & 248 & $\cong 650$ & 4.45 & 715 \\
\hline Tumba 12 & 220 & $600<\mathrm{T}<700$ & 3.59 & 610 \\
\hline Tumba 255 & 205 & 400 & 3.27 & 520 \\
\hline
\end{tabular}

\begin{tabular}{|l|c|c|c|c|}
\hline Parte del cuerpo & $\begin{array}{c}\text { Tamaño medio } \\
\text { de los cristales/( } \AA \text { ) }\end{array}$ & $\begin{array}{c}\text { Temperatura/DRX } \\
\text { (Piga et al, 2009)/ C }\end{array}$ & $\begin{array}{c}\text { Splitting Factor } \\
(\text { SF)-FTIR }\end{array}$ & $\begin{array}{c}\text { Temperatura/FTIR } \\
\text { (Piga et al, 2010)/C }\end{array}$ \\
\hline Cráneo & $>1500(1520)$ & $900<T<1000$ & 7,32 & 1000 \\
\hline Mandíbula & $>1500(2297)$ & 1000 & 6,18 & $>900$ \\
\hline Mandíbula(azul) & 230 & 700 & 4,32 & $700<\mathrm{T}<800$ \\
\hline Costilla & $>1500(2306)$ & 1000 & 6,93 & 1000 \\
\hline Costilla (azul) & 224 & 700 & 4,30 & $700<\mathrm{T}<800$ \\
\hline Vértebra 1 & $>1500(2200)$ & 1000 & 6,66 & Circa 900 \\
\hline Vértebra 2 & $>1500(1940)$ & 1000 & 6,52 & $800<\mathrm{T}<900$ \\
\hline Vértebra 3 & $>1500(1900)$ & 1000 & 7,21 & 1000 \\
\hline Vértebra 4 & $>1500(2248)$ & 1000 & 8,08 & 1000 \\
\hline Vértebra 5 & $>1500(2303)$ & 1000 & 6,91 & 1000 \\
\hline Cúbito derecho & $>1500(2079)$ & 1000 & 7,08 & 1000 \\
\hline Cúbito izquierdo & $>1500(1724)$ & 1000 & 6,67 & 900 \\
\hline Fémur derecho & $>1500(2316)$ & 1000 & 6,86 & 900 \\
\hline Fémur izquierdo & $>1500(1480)$ & $900<T<1000$ & 5,58 & $800<T<900$ \\
\hline Tibia izquierda & $>1500(1364)$ & 900 & 6,08 & $>800$ \\
\hline Tibia derecha & $>1500(1412)$ & 900 & 6,68 & 900 \\
\hline
\end{tabular}

Tabla 3.

Tamaño de los microcristales de hidroxiapatita, Splitting Factor y la estimación de las temperaturas calculadas con las dos técnicas de espectrometría. Ambas técnicas ofrecen resultados concordantes. 
La Tabla 2 muestra en detalle los resultados obtenidos mediante las técnicas de difracción de rayos X / FT-IR, que muestran un acuerdo sustancial entre ellos. Las diferencias que no superan los $120^{\circ} \mathrm{C}$ no son tan importantes como afectar a la validez de las dos técnicas, teniendo en cuenta las diferencias en la base químico-física de las teorías elaboradas.

Del análisis de estos resultados, se puede evidenciar la posibilidad de que los ritos funerarios púnicos de inhumación del Monte Sirai fueran precedidos de una combustión parcial de los cuerpos que pretendería quizás eliminar de forma rápida las partes blandas. Con toda probabilidad el fuego se interrumpiría después de pocos minutos (18 minutos, máximo 36) y los cuerpos (normalmente 2 por tumba) aún íntegros eran depositados en posición primaria uno encima del otro. También podría interpretarse como una combustión superficial mediante la quema de materiales vegetales sobre el mismo cuerpo, lo cual estaría en consonancia con la posición primaria de los cuerpos y la mayor temperatura alcanzada en la parte superior del hueso.

Sin embargo, el ritual en cuestión supone no pocos problemas puesto que, a pesar de que los cuerpos se encontraron en posición primaria, la fosa que los contenía, practicada en el tufo volcánico, no conservaba la más mínima traza de combustión. Merece la pena destacar que en el mismo yacimiento se identificó la zona dedicada al "ustrinum" o zona dedicada a la cremación de los cuerpos, pero es preciso añadir que a menudo en el interior de la misma sepultura se han encontrado dos cuerpos superpuestos, ambos en idéntico estado de semicombustión. Así pues, en el caso de Monte Sirai parece que se trata de un ritual nuevo, cuya práctica fue limitada en el tiempo y practicada quizás sólo alrededor del año 500 a.C. En la literatura antropológica no se han encontrado ejemplos similares en las necrópolis del mundo fenicio ni púnico ${ }^{22}$. Del mismo modo, la aplicación de estos mismos métodos a casos como el del cráneo mostrado en la Figura 1, indicaría sin lugar a dudas su exposición al fuego, a pesar de su apariencia no alterada.

Otra aplicación importante se refiere a la tumba de incineración 252. El descubrimiento excepcional de casi todas las partes del esqueleto nos ha permitido de realizar un análisis detallado para analizar la homogeneidad de la temperatura y la intensidad del fuego en diversas partes representante de todo el cuerpo.

Este tipo de análisis no es habitual pues es prácticamente imposible en tales contextos recuperar restos "in situ" de todo el esqueleto. Dado el excepcional estado de conservación de los diversos fragmentos (Figura 7), fue posible evaluar la eventual uniformidad de la temperatura en muestras representativas de todo el cuerpo.
La Tabla 3 muestra en detalle los resultados obtenidos mediante las técnicas de DRX/FT-IR. Los datos obtenidos con las dos técnicas son prácticamente concordantes, salvo en unos pocos casos (vértebras 1 y 2, cúbito izquierdo y ambos fémures) en los que las temperaturas obtenidas con el análisis de difracción de rayos $X$ son ligeramente más elevados. Estas diferencias no exceden los $100^{\circ} \mathrm{C}$ y no son significativas.

Los cambios de coloración y textura de los huesos así como los análisis de DRX/FT-IR sugieren una cremación a elevada temperatura, aproximadamente a $1000{ }^{\circ} \mathrm{C}$, aunque algunos fragmentos de coloración azulada sufrieron una temperatura menor entre $700 \mathrm{y}$ $800^{\circ} \mathrm{C}$.

De ambos tipos de análisis sobresale además el hecho que el calor debió afectar de forma bastante homogénea a lo largo del esqueleto, con lo que la pira debió ser alimentada en su conjunto, sin que existiera un centro principal.

\section{Conclusiones}

En las últimas décadas, la investigación y la experiencia de casos han aumentado en gran medida la capacidad de reconocer e interpretar los restos óseos quemados. Sin embargo, son necesarios nuevos métodos experimentales para aclarar la variedad de factores que conducen la gran cantidad de efectos térmicos.

La difracción de rayos $\mathrm{X}$ y análisis de FT-IR tienen varias ventajas significativas para la identificación de una cremación y para la estimación de la temperatura y la duración de un evento de cremación forense, centrándose en los cambios microscópicos en el hueso.

Los importantes avances en hardware han mejorado mucho la velocidad y la accesibilidad de DRX. Sin duda, estas técnicas de análisis tendrán un papel cada vez más importante en el campo de la ciencia forense en el futuro.

\section{Agradecimientos}

Los autores quieren agradecer al Prof. Stefano Enzo, Dr. Michele Guirguis (Universidad de Sassari, Italia), Dr. Massimo Piccinini (Porto Conte Ricerche, Alghero, Italia) y Dr. Ignasi Galtès su ayuda. Este trabajo ha sido posible gracias al proyecto "Giovani Ricercatori" 
de la Regione Autonoma della Sardegna, titulado: Studio archeometrico, antropologico e paleogenetico del materiale archeologico appartenente al sito fenicio-punico di Monte Sirai (Carbonia), y a los proyectos del MEC, CGL2008-800800/BOS y PR2009-0128.

\section{Bibliografía}

1. Symes SA, Rainwater CW, Chapman EN, Gipson DR, Piper AL. Patterned Thermal Destruction of Human Remains in a Forensic Setting. In: CW Schmidt, S.A. Symes (eds): The Analysis of Burned Human Remains. London: Academic Press, 2008;15-54.

2. Trancho GJ. Análisis antropológico de las necrópolis de cremación. Rev. Esp. Antr. Fís. 2010;31: 205-32.

3. Piga G, Hernández-Gasch JH, Malgosa A, Ganadu $\mathrm{ML}$, Enzo S. Cremation practices coexisting at the "S'Illot des Porros" Necropolis during the Second Iron Age in the Balearic Islands (Spain). Homo 2010;61:440-52.

4. Dehaan JD. Kirk's Fire Investigation. $5^{\text {th }}$ ed. Brady Publishing. New Jersey: Upper Saddle River, 2002.

5. Markiewicz D.A: Few takers for fake cremains; GBI seeks 'closure' in crematory scandal; families conflicted, The Atlanta Journal-Constitution Metro News, August 10 2005, 1B pp.

6. Subira M.E., Malgosa A: The Effect of Cremation on the Study of Trace Elements. Int. J. Osteoarchaeol. 1993;3:115-8.

7. Etxeberria F. Aspectos macroscópicos del hueso sometido al fuego. Revisión de las cremaciones descritas en el País Vasco desde la Arqueología. Munibe 1994;46:111-6.

8. Guillon F. Brules frais ou brules secs? Anthropologie Physique et Archéologie. París: Ed. C.N.R.S. 1987, 191-3.

9. Thompson TJU. Heat-induced dimensional changes in bone and their consequences for forensic anthropology. J. Forensic Sci. 2005;50(5):1008-15.

10. Paschalis EP, Betts F, DiCarlo E, Mendelsohn R, Boskey AL. FTIR Microspectroscopic analysis of normal human cortical and trabecular bone. Calcif. Tissue Int. 1997;61:480-6.

11. Thompson TJU, Islam M, Piduru K, Marcel A. An investigation into the internal and external variables acting on crystallinity index using Fourier Transform Infrared Spectroscopy on unaltered and burned bone. Palaeogeogr. Palaeoclimateol, Palaeoecol. 2011; 299: 168-174.
12. Wang XY, Zuo Y, Huang D, Hou X-D, Li YB: Comparative study of inorganic composition and crystallographic properties of cortical and cancellous bone. Biomed. Environ. Sci. 2010;23:473-480.

13. Nakano $T$, Tokumura A, Umakoshi Y. Variation in crystallinity of hydroxyapatite and the related calcium phosphates by mechanical grinding and subsequent heat treatment. Metall. Mater. Trans. 2002;A33:521-5.

14. Piga G, Thompson TJU, Malgosa A, Enzo S. The potential of X-Ray Diffraction (XRD) in the analysis of burned remains from forensic contexts. J. Forensic Sci. 2009;54(3):534-539.

15. Squires KE, Thompson TJU, Islam M, Chamberlain A. The application of histomorphometry and Fourier Transform Infrared Spectroscopy to the analysis of early Anglo-Saxon burned bone. J. Archaeol. Sci. 2011;38:2399-409.

16. Lutterotti L, Bortolotti M: Object oriented programming and fast computation techniques in MAUD, a program for powder diffraction analysis written in java. IUCr: Compcomm Newsletter 2003;1:43-50.

17. Bonucci E, Graziani G: Comparative thermogravimetric $X$-ray diffraction and electron microscope investigations of burnt bones from recent, ancient and prehistoric age. Atti della accademia Nazionale dei Lincei, Rendiconti, classe di scienze fisiche, matematiche e naturali 1975;59:517-32.

18. Shipman P, Foster G, Schoeninger M: Burnt bones and teeth: an experimental study of color, morphology, crystal structure and shrinkage. J. Archaeol. Sci. 1984;11:307-25.

19. Bohnert M, Rost T, Pollak S. The degree of destruction of human bodies in relation to the duration of the fire. Forensic Sci. Int. 1998;95:11-21.

20. Shemesh A. Crystallinity and diagenesis of sedimentary apatites. Geochim. Cosmochim. Acta 1990;54:2433-8.

21. Weiner S, Bar-Yosef O. States of preservation of bones from prehistoric sites in the Near East: a survey. J. Archaeol. Sci. 1990;17:187-96.

22. Piga G, Guirguis M, Bartoloni P, Malgosa A, Enzo S. A funerary rite study of the Phoenician-Punic Necropolis of Mount Sirai (Sardinia, Italy). Int. J. Osteoarchaeol. 2010;20:144-57. 